\title{
The bacterial cell cycle, chromosome inheritance and cell growth
}

Rodrigo Reyes-Lamothe ${ }^{1}$ and David J. Sherratt ${ }^{2}$

${ }^{1}$ Dept of Biology, McGill University, Montreal, Canada; ${ }^{2}$ Department of Biochemistry, University of Oxford, OX1 3QU, UK

rodrigo.reyes@mcgill.ca

david.sherratt@bioch.ox.ac.uk

correspondence to R.R.-L. and D.J.S.

\begin{abstract}
All viable bacterial cells, whether they divide symmetrically or asymmetrically, must coordinate their growth, division, cell volume and shape with 'chromosome inheritance', the processes that together maintain genome integrity over generations as chromosomes duplicate and segregate during each cell cycle. These processes include the organisation of DNA into nucleoids, controlled and faithful DNA replication, chromosome unlinking and faithful segregation into daughter cells. Chromosome inheritance also influences the timing and activity of physiological processes that lead to growth and cell division. We highlight the applications of quantitative single-cell studies that are revolutionising our mechanistic understanding of the cell cycle. Furthermore, we address how the study of a growing number of bacterial species enable the search for common principles that underlie chromosome inheritance coordinated with the cell cycle.
\end{abstract}

\section{[H1] Introduction}

Bacterial diversity is enormous, with the cellular volumes of different bacterial species spanning over seven orders of magnitude ${ }^{1}$ and genome size spanning two orders of magnitude [from $\sim 0.11 \mathrm{Mbp}$ in the obligate symbiont Candidatus Nasuia deltocephalinicola, to $\sim 15 \mathrm{Mbp}$ in the myxobacterium Sorangium cellulosum ${ }^{2-4}$ ]. Yet, all bacterial species maintain a characteristic cell volume and shape over generations, which requires the coordination of the processes that determine these phenotypes with inheritance of the genome in the form of the chromosome(s). Some bacteria produce multiple differentiated states either through asymmetric cell divisions and/or by morphological changes induced in response to changing environmental conditions. The ability to undergo and survive these transitions also has to be encoded in the genome. Feedback systems between the genome and other cellular factors influence each other - as observed, for example, in the interaction between metabolism and gene expression.

Unlike eukaryotes, many bacteria do not have distinct cell cycle. Consequently, checkpoints that act to prevent inopportune shift to the next cell cycle phase appear not to be generally present. Historically, the period from initiation of DNA replication of a bacterial chromosome to completion of its replication is defined as the C-period, which can be compared with the S-phase of eukaryotes, the period during which replication of all chromosomes occurs. The period from completion of replication to bacterial division is called the D-period, while that from birth to initiation of chromosome replication is the Bperiod (FIG. 1). The bacterial chromosome is organised by a $\sim 10^{3}$-fold linear compaction into a structure called the nucleoid, which contains DNA and DNA-binding proteins. Cytologically, 
the nucleoid may appear as a discrete compact structure with cytoplasm and polyribosomes between it and the inner membrane, or as a more amorphous form that appears to fill the whole cellular volume. Examples of the former are the nucleoids of Escherichia coli and Bacillus subtilis, and of the latter, Caulobacter crescentus. In the former case, the compact structure is at least partly maintained by molecular crowding caused by the huge number of polyribosomes and their associated proteins ${ }^{5}$, because polyribosome disruption after addition of the transcriptional inhibitor, rifampicin, leads to a decompacted nucleoid filling the whole cell ${ }^{6}$. Duplication of the long chromosomal DNA molecules starts at a single sequence-specific point, the origin of replication. DNA synthesis proceeds bidirectionally from this point, and takes typically tens of minutes for completion in model bacteria grown in the laboratory. Segregation of chromosomal DNA into the developing daughter cells occurs in parallel to DNA replication. In rod-shaped bacteria, segregation begins with the movement of the replication origin to symmetrical positions on the two halves of the long axis of the cell - near the one quarter and three-quarter positions, or at the cell poles. This is followed by the movement of replicated loci to cell locations that depend on the position of the replication origin, and ultimately result in one of two possible types of nucleoid folding (FIG. 2). Cell division normally initiates in nucleoid-free areas when chromosomes have been segregated. It starts by the formation of a positioned Z-ring, formed by the bacterial tubulin FtsZ, and other cell division proteins, that act along with membrane invagination and cell wall biosynthetic enzymes to complete cell division.

Recent technological advances have enabled DNA inheritance, cell growth and cell division events to be followed at high throughput and with great precision over generations in single bacterial cells. Genetic loci and molecular machines can be imaged with unprecedented spatial and temporal resolution. These advances, and complementary theoretical simulations and modelling studies, have 're-ignited' interest in the coordination of events that relate directly or indirectly to the bacterial cell cycle (BOX 1). We refer the reader to excellent recent reviews that describe the mechanisms for DNA replication, chromosome segregation ${ }^{7-9}$, and cell division ${ }^{10}$, and that critically discuss the complexities and state of play that have emerged from recent studies of cell volume control and its coordination with those cellular events ${ }^{1,11}$.

In this Review, we describe the intimate links between the chromosome and the cell cycle, taking a DNA-centric view of how cells coordinate their DNA inheritance with cell growth and division. We first explore the principles of how the cell cycle is coupled to cell growth and cell volume, and we describe the contributions of chromosome structure and nucleoid folding to cell cycle regulation. Moreover, we detail the cellular processes involved in the initiation of DNA replication and DNA segregation and explore how those processes are linked to cell growth and cell division.

\section{[H1] Cell volume, growth and DNA replication}

Pioneering ensemble studies of the Escherichia coli cell cycle in the period 1958-1968 provided important insights into the bacterial cell cycle in a model organism in which the generation time can be shorter than the time it takes to replicate its chromosome ${ }^{12-14}$ (reviewed in ${ }^{1,11}$ ). Therefore, overlapping rounds of replication are required to achieve a constant DNA content/cell over generations, with new born cells having partially replicated chromosomes at birth (FIG. 1). Three visionary inter-related principles emerged from this early work. First, a growth law proposed that bacterial cell volume depends exponentially on growth rate and increases exponentially as growth proceeds ${ }^{12}$. Second, during growth in 
which the generation time is shorter than the replication time, the combined time of C and D periods is constant and independent of growth rate ${ }^{13}$. Third, in populations of cells, initiation of DNA replication occurs at a fixed cell volume per origin ${ }^{14}$. Fast-growing bacteria that include E. coli, Bacillus subtilis and Vibrio species have all evolved to coordinate overlapping replication cycles with cell growth, chromosome segregation and subsequent cell division. By contrast, slower growing bacterial species (for example, the $\alpha$-proteobacteria Caulobacter crescentus and Rhodobacter sphaeroides), and potentially fast-growing bacteria in nutrientlimited conditions, complete replication in the same generation as it was initiated ${ }^{15-18}$, as do characterized Archaea ${ }^{19}$ (FIG. 1).

Although, each of the three principles, when applied to bacterial populations, has largely stood the test of time, mechanistic insight into the processes that underlie these observations has been elusive. Nevertheless, the advances in imaging alluded to above have provided important phenomenological information at the single-cell level, which is providing a rich platform for discussion of the possible biological processes and controls responsible for the observed behaviour (BOX 1). Studies examining cell volume, growth properties and DNA replication in steady state and perturbed conditions ${ }^{20-22}$ have resulted in multiple predictions relating to the coordination between the cellular events and the genome. Among them is the demonstration from most studies that initiation of $E$. coli DNA replication occurs at a fixed cell volume/ori, independent of birth size and growth rate in individual cells ${ }^{20-22}$, in-line with Donachie's original paper, suggesting that replication initiation is a reset point in the cell cycle. Initiation of replication is therefore likely to be crucial during cell cycle control, but other aspects of the genome, such as gene content, orientation and arrangement, as well as chromosome architecture (see below), also have important contributions.

\section{[H1] The structure of chromosomes and nucleoids}

Bacteria have evolved to thrive in specific environmental niches. Genome content and organisation provide the platform for determining the nature of the cell cycle and its regulation. As such, the genomes of many bacteria have not evolved for fast growth, but rather to survive and proliferate in their specific niche(s), with some bacteria having evolved to inhabit at least two very different niches. Gene content defines the potential for metabolic versatility and the capacity to generate energy and extract the essential compounds from the environment needed for growth and proliferation. Metabolic diversity generates a wide range of generation times among species, ranging from few minutes in the common model bacteria used in the lab to thousands of years such as in bacteria living in deep sub-floor sediments 23,24 .

In addition to the gene content, other traits of the chromosomes, like the arrangement and orientation of genes, play important roles for the coordination of genome inheritance with the cell cycle. Characterized bacteria have a single or multiple gene-dense chromosome(s), with each having a single replication origin. The arrangement and orientation of genes in the chromosome influence cell physiology, by facilitating genome duplication and by regulating the expression level of genes. There is a strong bias for essential genes regardless of their expression level - to be coded in the leading strand. As replication and transcription occur simultaneously, the strand bias presumably avoids head-to-head collisions

of RNA polymerases with the replisome which could lead to genome instability ${ }^{25-28}$. In addition, cells exploit the positioning of genes relative to the replication origin to modulate their relative gene dosage in actively replicating cells. This effect is particularly strong in fast growing bacteria, where the generation time is shorter than the time needed for 
chromosome synthesis, and origin-proximal genes can be present in eight or more copies per cell during active growth. Indeed, a predicting feature of fast-growing bacteria is the positioning of genes involved in protein synthesis and their copy number in the genome. The RNA polymerase, ribosomal RNA genes and ribosomal protein genes in fast-growing bacteria are all positioned near the replication origin (FIG. 2). Given that the rate of cell growth is correlated to the concentration of ribosomes in the cell ${ }^{29}$, the resulting transient gene amplification allows cells to bypass the limitation imposed by the rates of transcription in the production of proteins, and hence maximizes proliferative growth ${ }^{30}$.

The position and orientation of short non-coding sequences in the chromosomes also help to direct the cell cycle (reviewed in ${ }^{31}$ ). Taking $E$. coli as an example, there are multiple sites required to avoid over-replication near the place where replication forks encounter (ter sites); and for chromosome segregation (KOPS sequences). A site, datA, situated near oriC binds the ATP-form of the replication initiation protein DnaA and stimulates ATP hydrolysis (described later). GATC sequences, that become hemimethylated for a short time after replication, act to prevent premature replication initiations - via binding to the SeqA protein - as well as directing mismatch repair to the newly synthesized DNA strand. Chi sequences stimulate and direct homologous recombination initiated by double-strand breaks. Finally, sequences that bind the nucleoid occlusion factor SImA are responsible for ensuring cell constriction is initiated once the bulk of chromosomal DNA has been segregated. This is an incomplete list that include many other short sequences, and their cognate proteins, having other roles in the cell.

Inside the cell, chromosomes are compacted $~ 1000$ times linearly into the nucleoid. DNA supercoiling, molecular crowding and DNA-binding proteins contribute to this compaction ${ }^{32}$. Simple topological considerations predict that chromosomes must be organised into looped structures, rather than having the DNA wrapped into a ball. Such chromosomal loops have been visualised in prokaryotes and eukaryotes 33,34 . They form independent supercoiled topological domains, which are organized such that genes have approximate addresses in the bacterial nucleoid that change predictably during replication ${ }^{8,35-39}$. Furthermore, the replichores have specific cellular arrangements, depending on the bacterial species and cell physiology (FIG. 2). It now appears that SMC complexes have key roles in organising this loop structure through 'loop extrusion' ${ }^{40,41}$, while bacterial NAPs probably stabilise such structures. A higher order 'macrodomain' organisation, which is particularly evident for the replication termination region (ter), has also been revealed in E. coli ${ }^{42}$. Indeed, the E. coli 0.7 Mbp ter macrodomain contains multiple matS sites, which bind MatP, and this interaction is responsible for specifying the ter macrodomain. Binding of MatP to matS leads to depletion of the SMC complex MukBEF from ter, which results in an altered organization 43,44 . Compaction and organisation of chromosomes facilitate their partition before cell division and regulate cell volume, as evidenced by the increased anucleated cell formation in mutants affecting these processes, such as in the case of Muk mutants ${ }^{45}$.

\section{[H1] Replication initiation}

Commitment to genome duplication is arguably the most important event in the cell cycle. Initiation of DNA replication represents a Rubicon cross-commitment of cells to DNA elongation, once initiated DNA replication must reach completion, taking up a significant fraction of the cell cycle. In most bacteria, only one initiation event occurs per cell generation 
in steady state conditions; exceptions may include polyploid bacteria (see section below on cyanobacteria). Initiation occurs at a specific site of the chromosome, thus controlling the region in which pairs of replication forks will meet. Multiple predictions have been made on the regulation of initiation based on studies on the cell cycle 20-22; here we highlight two of them. First, initiation occurs at a fixed volume per origin, marking the start of the cell cycle. Second, the relation between volume increase and initiating capacity during growth makes models for initiation incompatible with a mechanism where regulation occur only through changes in the copy number of proteins, including the initiator. It requires other type of mechanisms that can sense exquisitely small changes in cell volume. These predictions are interrelated, and are also linked to the growth law, but it is still uncertain what are the molecular mechanisms driving the regulation of initiation. Based on the current knowledge on initiation from multiple organisms, we highlight below the role of DnaA-ATP filament growth as a key element in the still partially-understood regulation of initiation.

\section{[H2] Replication origin melting.}

DNA replication initiates with the loading of the ring-shaped helicase at the replication origin. The conserved initiator protein DnaA mediates DNA melting and the recruitment of the helicase and its loader to the bacterial chromosome, or to the primary chromosome in species with multiple chromosomes (see below). Like other AAA+ proteins, multiple copies of DnaA oligomerize in an ATP-dependent manner, forming a helical structure ${ }^{46}$. The consensus binding sequence for the DnaA protein in most bacteria is a highly-conserved asymmetric nine-nucleotide long DNA motif (DnaA box). Replication origins contain multiple copies of DnaA boxes positioned close to an AT-rich DNA unwinding element (DUE) ${ }^{47}$. The replication origins vary in sequence, length and structure in different organisms ${ }^{47}$. It is widely thought that the arrangement of DnaA boxes in the replication origin promotes the formation of DnaA filaments around DNA, exerting helical torsion as it grows on DNA, resulting in the unwinding of the DUE. However, this model has recently been challenged by the observation that unwinding of the DUE can be achieved in the absence of DnaA-ATP or DnaA filaments in a synthetically engineered oriC ${ }^{48}$, suggesting that DnaA oligomerization is important for fulloccupancy of DnaA at oriC, but that any deformation resulting from filament growth is dispensable for DNA melting.

\section{[H2] Timing initiation of DNA replication.}

What determines when origins fire? Reducing the prototypical $E$. coli system to its bare principles shows that DnaA must be in its DnaA-ATP state, and that the weak-binding DnaA boxes in the replication origin have to be fully-occupied to unwind the DUE. The replication origin in E. coli contains three strong-binding DnaA boxes (R1, R2 and R4) that flank two sets of four weak-binding boxes (R5M, $22,11-13$, and C1-C3) ${ }^{49-51}$, although only a subset of these boxes are essential for activity ${ }^{52,53}$. The strong-binding boxes are occupied throughout the cell cycle and bind DnaA in both of its nucleotide-binding states. These sites serve as nucleation points for DnaA oligomerization and also to suppress the intrinsic capacity of DUE to unwind prematurely ${ }^{54,55}$. DnaA in E. coli binds ATP tightly, but only weak has weak ATPase activity on its own. The fraction of DnaA bound by ATP fluctuates over the cell cycle ${ }^{56}$, defining periods of high initiating activity by DnaA, accompanied by occupancy of weakbinding sites and formation of DnaA-ATP filaments ${ }^{48-50}$ (FIG. 3). The Hda protein and the datA locus independently mediate the increase in the DnaA ATPase activity after replication initiation, thereby preventing immediate re-initiation ${ }^{57,58}$. Other mechanisms stimulate the 
accumulation of DnaA-ATP later in the cell cycle, through DARS loci and interaction with phospholipids ${ }^{59,60}$. Hundreds of DnaA boxes distributed across the genome increasingly capture free copies of DnaA as the genome duplicates, but this process plays only a modest effect as timing of initiation is insensitive to moderate concentration changes in DnaA ${ }^{61}$. In addition, accessory proteins, SeqA, IHF, Fis and DiaA, all modulate binding of DnaA at the weak DnaA boxes, thereby fine-tuning initiation time. SeqA prevents rebinding of DnaA at weak-binding boxes, hence subsequent re-initiations at the same origin, by binding to hemimethylated GATC sites for about 10 minutes after origin firing ${ }^{62-64}$. We refer to multiple recent reviews on initiation for the details on this process ${ }^{65-67}$.

Studies of initiation timing in other bacteria reveal that limiting DnaA filament growth is a conserved strategy, although the mechanisms by which this is mediated vary. For example, B. subtilis exploits the use of protein partners to prevent the formation of DnaA filaments. Nucleotide-binding by DnaA in this organism is weaker than in $E$. coli - at least in vitro - resulting in almost 10-fold faster exchange with a half-life of 5 minutes ${ }^{68}$, suggesting a relatively invariant ratio of ATP:ADP bound to DnaA, and explaining a lack of reported mechanisms to activate its ATPase. Three different systems limit the filament growth of DnaA by direct binding to this protein during active cell growth: the initiation factor DnaD ${ }^{69}$; the beta-clamp binding protein YabA ${ }^{69}$; and the partition system component Soj ${ }^{70}$. In $C$. crescentus, DNA replication has to align with its two differentiation states ${ }^{16}$. DNA replication only occurs in stalked cells. Similar to $E$. coli, timing of initiation seems to largely depend on the regulation of the nucleotide state of DnaA, which is at least partly regulated by an Hda orthologue, $\mathrm{HdaA}{ }^{71-73}$. Repression of DNA replication in swarmer cells is mediated by the protein CtrA, which binds the replication origin and inhibits DnaA binding shortly after initiation. In replicating stalked cells, CtrA copy number fluctuates during the cell cycle, but the timing of initiation is only modestly affected by $\operatorname{CtrA}{ }^{73,74}$.

\section{[H2] Regulation of initiation in cells with multiple chromosomes.}

About $10 \%$ of characterized bacteria carry additional smaller secondary chromosomes, which have clearly evolved from low-copy plasmids ${ }^{75}$. Plasmids, regardless of length and copy number, initiate stochastically with respect to the start of the cell cycle, but maintain a fixed copy number per cell volume ${ }^{76,77}$. In contrast to plasmids, secondary chromosomes have acquired mechanisms to synchronize their duplication with the primary chromosome. Proteobacteria with multipartite genomes, including Vibrio cholerae, Burkholderia cenocepacia, Sinorhizobium meliloti and Brucella abortus, initiate DNA replication sequentially, starting with the primary chromosome and followed, typically with a delay of tens of minutes, by the secondary chromosome(s) ${ }^{78-81}$. In $V$. cholerae, currently the best understood system, initiation at its primary chromosome indirectly controls the secondary chromosome ${ }^{82}$. Initiation of the primary chromosome is controlled by DnaA and accessory initiation proteins shared with its relative, E. coli. Initiation of the secondary chromosome is instead reminiscent to that of iteron plasmids and uses a specific initiator called RctB 83,84 . This protein becomes competent for initiation as a chromosomal locus - called ctrS - in the primary chromosome duplicates ${ }^{82} 85$. Hence, it is the synchrony in termination of the two chromosomes, and not in their initiation, which is important, perhaps because this ensures that segregation of both chromosomes is timely with respect to cell division ${ }^{78}$.

Yet another genome management strategy in bacteria is polyploidy, the presence of multiple complete copies of the same chromosome in the cell. Ploidy in cyanobacteria ranges 
from one, as in E. coli and other model bacteria, to over twenty copies of the chromosome per cell ${ }^{86}$. In the case of the Synechococcus elongatus, which carries an average of 4 chromosomes ${ }^{86}$, DNA replication is both light-dependent and dependent on photosynthetic electron transport ${ }^{87,88}$, and occurs asynchronously among the chromosomes in the same cell, resulting in only one chromosome duplicating at a given time during exponential growth, hence breaking the rule of one replication event per cell cycle as multiple replication events are needed to duplicate the copy number ${ }^{88-90}$. It has been suggested that polyploidy and stochastic firing of replication, reminiscent to that of plasmids, may help to minimize gene dosage effects paired with the cell cycle on the circadian clock of cyanobacteria ${ }^{91}$. A linear relation between cell volume and number of chromosomes ${ }^{89,90}$, suggests that origin firing occurs after a fixed increase in cell volume as in other organisms, but that this organism has decoupled its replication and division cycles. Cell cycle control of DNA replication must apply, although likely allowing considerable error, in extreme cases of polyploidy, like in the firmicute Epulopiscium, which can carry tens of thousands of copies of its single chromosome, but maintains a linear relation between cell volume and chromosome copy number ${ }^{92}$.

\section{[H1] Replication elongation and termination}

Cell volume homeostasis requires not only that DNA replication initiates at a specific time during the cell cycle, but also that it progresses at an appropriate rate during the elongation stage. The maximal rate of elongation varies about one order of magnitude in characterized bacteria belonging to multiple taxa ${ }^{93,94}$. Individual organisms also modulate the rate of DNA synthesis according to their growth rate ${ }^{95,96}$ - although seemingly with a defined upper limit, as fast growing cells with overlapping cell cycles have almost constant $C$ and $D$ periods independent of growth rate ${ }^{1,16-18}$ (FIG. 1).

DNA replication is carried out by a multiprotein molecular machine, the replisome ${ }^{97,98}$ (FIG. 4). At replication initiation, DNA unwinding by the helicase at the replication origin subsequently recruits the rest of the replisome subunits. Directional unwinding of the antiparallel double strand DNA (dsDNA) by the helicase permits continuous elongation on one of the strands (leading strand). The second strand (lagging strand) requires that synthesis proceeds in Okazaki fragments of 1-2kbp. The replisome is a dynamic structure as most of its subunits, except for the helicase, every few seconds ${ }^{99-101}$. Despite some differences in composition, the majority of the proteins contained in the replisome are conserved across bacteria ${ }^{102}$. The mechanisms known to modulate the rate of DNA replication act by controlling the pool of dNTPs or by allosterically regulating primase activity. Changes in the activity of the ribonucleotide reductase (RNR), the enzyme that synthesizes dNTPs, coded by $n r d A B$ in $E$. coli in aerobic conditions, results in a slowdown of the replication rate when downregulated 10395 104, or in faster rates when this enzyme is overproduced ${ }^{105}$. Both of these perturbations are accompanied by changes in the average cell volume in a population. Activity of the RNR is regulated allosterically at the level of the enzyme. However, coordination with the cell cycle is achieved by regulation of gene expression. Expression from this operon peaks near the time of initiation, activates as response to inhibition of DNA replication, and is expected to vary according to growth rate - hence the different elongation rates ${ }^{106-108}$. DnaA acts as a transcription factor, binding to three sites in the $\operatorname{nrdAB}$ promoter. Regulation of expression by DnaA can explain its link to DNA replication, but so far it seems not to be linked to the timing of expression ${ }^{106}$. DnaA-ATP acts as an activator or inhibitor of expression when it is present at low or high concentration in the cell, respectively ${ }^{106}$. Consequently, an essential function of $\mathrm{Hda}$ in the cell is to prevent the repression of this promoter by excessive 
DnaA-ATP 109,110 . NrdR is a likely candidate to regulate the expression of $n r d A B$ according to the metabolic state of the cell ${ }^{111}$. This transcription factor oligomerizes in response to the phosphorylation state of the nucleoside it binds - it can bind to AMP, ADP and ATP ${ }^{112}$. Longer oligomers, incapable of repressing $n r d A B$ expression, are formed when bound to ATP ${ }^{112}$, suggesting that greater levels of expression occur at times when cells are metabolically more active.

A second known mechanism that modulates the rate of DNA synthesis acts by modulating primase activity. In response to starvation, synthesis of the signal molecules ppGpp and pppGpp inhibit primase activity in both $B$. subtillis and $E$. coli ${ }^{113}$. Inhibition is significantly stronger in $B$. subtillis, where DNA replication is halted within few minutes from exposure to amino acid starvation ${ }^{114}$. Drastic regulation in response to the metabolic state of cells is also observed in S. elongatus, where DNA synthesis is completely inhibited -by a still unknown mechanism - immediately after shifting cells from light to dark conditions ${ }^{87}$. Stalled replication forks are normally a substrate for DNA recombination and a signal for the SOS response - which has high mutagenic potential ${ }^{115}$. However, for still unclear reasons, halting the replication fork through the inhibition of primase does not induce DNA recombination, or the SOS response ${ }^{114}$.

Replication ends as two converging replication forks meet. Multiple factors act to ensure complete replication, prevent over-replication and preserve genome integrity, including the Ter-tus system in E. coli, and enzymes that act by preventing the formation of specific DNA structures, although the details of how this process occurs are still emerging ${ }^{116-}$ 118. These systems have no influence on cell cycle regulation as there is no evidence that replication termination itself provides a signal that initiates the beginning of the $D$ period. Rather, movement of chromosomal DNA away from the potential division site allows a functional divisome to form, and the timing at which this occurs, relative to replication termination, sets the length of the $D$ period.

\section{[H1] Chromosome unlinking and segregation}

Timely partition of the genome is a requisite for accurate timing of cell division. Despite its importance, evidence suggest that chromosome segregation is not a point of regulation for the cell cycle. As such, we only discuss them briefly, but refer to recent reviews on this subject $7,9,119$. Genome partition can be divided in two distinct problems, the resolution of topological links between the sister chromosomes, and the correct spatial positioning of chromosomes to ensure even distribution of the copies of the genome among sister cells. Nucleoid positioning is linked to cell division through nucleoid occlusion, which prevents the formation of the septum formation until chromosome segregation is completed. This regulation is likely due to the incapacity of the divisome to assemble properly, perhaps because there is insufficient space in the extranucleoid region, and may be enhanced by any membrane association of the nucleoid ${ }^{10}$. Nucleoid occlusion is also mediated through dedicated systems: in $E$. coli, SImA binds the chromosome other than in ter and inhibits FtsZ polymerisation. Similarly, in B. subtilis Noc binds DNA but is excluded from the ter domain, although in this case its membrane association prevents divisome formation ${ }^{120}$.

As a consequence of the inter-wound strands of duplex DNA and semi-conservative DNA replication, $\sim 10^{5}$ links in every megabase of chromosomal DNA need to be removed during every replication cycle (DNA topology and DNA replication is discussed in ${ }^{121}$ ). This can only be achieved by the action of topoisomerases. The two major topoisomerases that act in 
unlinking in most bacteria are DNA gyrase and topoisomerase IV, both type 2 topoisomerases 121. The relative contribution of these systems to the total unlinking burden remains to be determined. Decatenation of these physical linkages by topoisomerase IV influences the time between replication of a locus and its subsequent segregation ${ }^{122,123}$. DNA recombination events, followed by crossovers between sister chromosomes, result in the generation of chromosome dimers once every few generations. The XerCD-dif-FtsK dimer resolution system acts within the ter region to separate the covalently linked chromosomes dimers ${ }^{124}$. In addition, this system can also act locally in decatenation ${ }^{125,126}$, potentially helping in the last stages of chromosome segregation.

The elusive search for bacterial chromosome segregation systems has revealed one family of segregation systems, which were initially identified as being necessary for the segregation of large low copy plasmids. Such ParAB-parS systems are widespread, but not ubiquitous, and frequently they are not essential other than for secondary chromosomes and large plasmids, leading to the idea of entropic processes driving separation ${ }^{127}$. Additionally, the action of bacterial SMC proteins, including E. coli MukBEF, in organizing and individualising chromosomes is important for efficient chromosome segregation ${ }^{7,44}$. Like replication, segregation is progressive and sequential in those bacteria that have been investigated $8,35-$ 39 , with cohesion time being determined largely by the time for decatenation ${ }^{122,123}$. The DNA translocator FtsK and its orthologues, like SpollIE, are likely to be involved in late steps of chromosome segregation. These proteins act as a coordinator between chromosome segregation and cell division, by participating in both processes ${ }^{128}$.

\section{[H1] Conclusions and Perspective}

Multiple connections have been established between these processes, encompassing the structure of chromosomes and nucleoids, and the processes of DNA replication and segregation. It is expected that greater mechanistic detail in the description of these links, and a greater number of examples from non-model organisms will be reported in the future. A major outstanding question is how the timing of initiation is regulated. It is foreseeable that single-cell quantitative approaches in model and a growing number of non-traditionally studied bacteria will help answering this question.

In the wild, the conditions that permit steady-state growth of bacteria will most frequently last for short periods of time. The norm in most environments is for bacteria to experience near-starvation or stress conditions, which they must adapt to survive ${ }^{129}$. Hence, by necessity bacteria must adapt to survive through long periods of cell dormancy. These same strategies will also be important in biofilms, and the generation of bacterial persisters after exposure to antibiotics and other stresses. It follows that bacterial chromosomes must have evolved genes and processes to help in their survival during non-growth states. Although much remains to be explored in this area, some insight can be obtained from the available studies. For example, a striking difference is the regulation and role of DNA replication during quiescence, as coordination of the cell volume and chromosomal inheritance is unnecessary. In contrast to steady-state growth, the timing and location of origin firing is much more flexible in these situations. In some circumstances, even completion of DNA replication seems not to be the goal. Streptococcus pneumoniae responds to antibiotics targeting DNA replication by allowing continued initiation of DNA replication, with accompanying slow replication forks progression. This leads to increased gene dosage around the replication origin and consequent activation of competence, because competence genes are found close 
to the origin ${ }^{130}$. A different example is the dispensability of DnaA in at least some cyanobacteria, which is not only not detrimental for growth, but improves their survival during the stationary phase ${ }^{131}$.

A final area of interest for the future is the study of the evolutionary origin of the coordination between the chromosome and the cell cycle. Early cells are thought to have lacked a cell wall, which must have resulted in a variable volume and copy number of genomes per cell ${ }^{132}$. Study of many more diverse bacteria, some of which lack detectable peptidoglycans and cell division machinery, will help in the understanding of how coordination between chromosome 'management', growth and the cell cycle evolved and occurs mechanistically. 


\section{References}

1 Willis, L. \& Huang, K. C. Sizing up the bacterial cell cycle. Nature reviews 15, 606620, doi:10.1038/nrmicro.2017.79 (2017).

2 Schneiker, S. et al. Complete genome sequence of the myxobacterium Sorangium cellulosum. Nat Biotechnol 25, 1281-1289, doi:10.1038/nbt1354 (2007).

3 Han, K. et al. Extraordinary expansion of a Sorangium cellulosum genome from an alkaline milieu. Sci Rep 3, 2101, doi:10.1038/srep02101 (2013).

4 Bennett, G. M. \& Moran, N. A. Small, smaller, smallest: the origins and evolution of ancient dual symbioses in a Phloem-feeding insect. Genome Biol Evol 5, 1675-1688, doi:10.1093/gbe/evt118 (2013).

5 Jun, S. Chromosome, cell cycle, and entropy. Biophysical journal 108, 785-786, doi:10.1016/j.bpj.2014.12.032 (2015).

6 Stracy, M. et al. Live-cell superresolution microscopy reveals the organization of RNA polymerase in the bacterial nucleoid. Proceedings of the National Academy of Sciences of the United States of America 112, E4390-4399, doi:10.1073/pnas.1507592112 (2015).

7 Reyes-Lamothe, R., Nicolas, E. \& Sherratt, D. J. Chromosome Replication and Segregation in Bacteria. Annu Rev Genet, doi:10.1146/annurev-genet-110711-155421 (2012).

8 Wang, X., Montero Llopis, P. \& Rudner, D. Z. Bacillus subtilis chromosome organization oscillates between two distinct patterns. Proceedings of the National Academy of Sciences of the United States of America 111, 12877-12882, doi:10.1073/pnas.1407461111 (2014).

9 Badrinarayanan, A., Le, T. B. \& Laub, M. T. Bacterial chromosome organization and segregation. Annu Rev Cell Dev Biol 31, 171-199, doi:10.1146/annurev-cellbio100814-125211 (2015).

10 den Blaauwen, T. Prokaryotic cell division: flexible and diverse. Current opinion in microbiology 16, 738-744, doi:10.1016/j.mib.2013.09.002 (2013).

11 Sauls, J. T., Li, D. \& Jun, S. Adder and a coarse-grained approach to cell size homeostasis in bacteria. Current opinion in cell biology 38, 38-44, doi:10.1016/j.ceb.2016.02.004 (2016).

12 Schaechter, M., Bentzon, M. W. \& Maaloe, O. Synthesis of deoxyribonucleic acid during the division cycle of bacteria. Nature 183, 1207-1208 (1959).

13 Cooper, S. \& Helmstetter, C. E. Chromosome replication and the division cycle of Escherichia coli B/r. J Mol Biol 31, 519-540 (1968).

14 Donachie, W. D. Relationship between cell size and time of initiation of DNA replication. Nature 219, 1077-1079 (1968).

15 Dubarry, N., Willis, C. R., Ball, G., Lesterlin, C. \& Armitage, J. P. In Vivo Imaging of the Segregation of the 2 Chromosomes and the Cell Division Proteins of Rhodobacter sphaeroides Reveals an Unexpected Role for MipZ. MBio 10, doi:10.1128/mBio.02515-18 (2019).

16 Collier, J. Regulation of chromosomal replication in Caulobacter crescentus. Plasmid 67, 76-87, doi:10.1016/j.plasmid.2011.12.007 (2012).

17 Helmstetter, C., Cooper, S., Pierucci, O. \& Revelas, E. On the bacterial life sequence. Cold Spring Harb Symp Quant Biol 33, 809-822 (1968).

18 Stokke, C., Waldminghaus, T. \& Skarstad, K. Replication patterns and organization of replication forks in Vibrio cholerae. Microbiology 157, 695-708, doi:10.1099/mic.0.045112-0 (2011).

19 Lindas, A. C. \& Bernander, R. The cell cycle of archaea. Nature reviews 11, 627-638, doi:10.1038/nrmicro3077 (2013). 
20 Si, F. et al. Invariance of Initiation Mass and Predictability of Cell Size in Escherichia coli. Curr Biol 27, 1278-1287, doi:10.1016/j.cub.2017.03.022 (2017).

21 Zheng, H. et al. Interrogating the Escherichia coli cell cycle by cell dimension perturbations. Proceedings of the National Academy of Sciences of the United States of America 113, 15000-15005, doi:10.1073/pnas.1617932114 (2016).

22 Wallden, M., Fange, D., Lundius, E. G., Baltekin, O. \& Elf, J. The Synchronization of Replication and Division Cycles in Individual E. coli Cells. Cell 166, 729-739, doi:10.1016/j.cell.2016.06.052 (2016).

23 Whitman, W. B., Coleman, D. C. \& Wiebe, W. J. Prokaryotes: the unseen majority. Proceedings of the National Academy of Sciences of the United States of America 95, 6578-6583 (1998).

24 Lomstein, B. A., Langerhuus, A. T., D'Hondt, S., Jorgensen, B. B. \& Spivack, A. J. Endospore abundance, microbial growth and necromass turnover in deep sub-seafloor sediment. Nature 484, 101-104, doi:10.1038/nature10905 (2012).

25 Rocha, E. P. \& Danchin, A. Essentiality, not expressiveness, drives gene-strand bias in bacteria. Nat Genet 34, 377-378, doi:10.1038/ng1209 (2003).

26 Rocha, E. P. \& Danchin, A. Gene essentiality determines chromosome organisation in bacteria. Nucleic acids research 31, 6570-6577 (2003).

27 De Septenville, A. L., Duigou, S., Boubakri, H. \& Michel, B. Replication fork reversal after replication-transcription collision. PLoS Genet 8, e1002622, doi:10.1371/journal.pgen.1002622 (2012).

28 Wang, J. D., Berkmen, M. B. \& Grossman, A. D. Genome-wide coorientation of replication and transcription reduces adverse effects on replication in Bacillus subtilis. Proceedings of the National Academy of Sciences of the United States of America 104, 5608-5613, doi:10.1073/pnas.0608999104 (2007).

29 Bremer, H. \& Dennis, P. P. Modulation of Chemical Composition and Other Parameters of the Cell at Different Exponential Growth Rates. EcoSal Plus 3, doi:10.1128/ecosal.5.2.3 (2008).

30 Couturier, E. \& Rocha, E. P. Replication-associated gene dosage effects shape the genomes of fast-growing bacteria but only for transcription and translation genes. $\mathrm{Mol}$ Microbiol 59, 1506-1518, doi:10.1111/j.1365-2958.2006.05046.x (2006).

31 Touzain, F., Petit, M. A., Schbath, S. \& El Karoui, M. DNA motifs that sculpt the bacterial chromosome. Nature reviews 9, 15-26, doi:10.1038/nrmicro2477 (2011).

32 Woldringh, C. L. \& Nanninga, N. Structural and physical aspects of bacterial chromosome segregation. Journal of structural biology 156, 273-283 (2006).

33 Paulson, J. R. \& Laemmli, U. K. The structure of histone-depleted metaphase chromosomes. Cell 12, 817-828 (1977).

34 Kavenoff, R. \& Bowen, B. C. Electron microscopy of membrane-free folded chromosomes from Escherichia coli. Chromosoma 59, 89-101 (1976).

35 Wang, X., Possoz, C. \& Sherratt, D. J. Dancing around the divisome: asymmetric chromosme segregation in Escherichia coli. Genes \& development 19, 2367-2377 (2005).

36 Nielsen, H. J., Li, Y., Youngren, B., Hansen, F. G. \& Austin, S. Progressive segregation of the Escherichia coli chromosome. Mol Microbiol 61, 383-393 (2006).

37 Viollier, P. H. et al. Rapid and sequential movement of individual chromosomal loci to specific subcellular locations during bacterial DNA replication. Proceedings of the National Academy of Sciences of the United States of America 101, 9257-9262 (2004).

38 Vallet-Gely, I. \& Boccard, F. Chromosomal organization and segregation in Pseudomonas aeruginosa. PLoS Genet 9, e1003492, doi:10.1371/journal.pgen.1003492 (2013). 
39 David, A. et al. The two Cis-acting sites, parS1 and oriC1, contribute to the longitudinal organisation of Vibrio cholerae chromosome I. PLoS Genet 10, e1004448, doi:10.1371/journal.pgen.1004448 (2014).

40 Nasmyth, K. \& Haering, C. H. The structure and function of SMC and kleisin complexes. Annual review of biochemistry 74, 595-648, doi:10.1146/annurev.biochem.74.082803.133219 (2005).

41 Ganji, M. et al. Real-time imaging of DNA loop extrusion by condensin. Science 360, 102-105, doi:10.1126/science.aar7831 (2018).

42 Mercier, R. et al. The MatP/matS site-specific system organizes the terminus region of the E. coli chromosome into a macrodomain. Cell 135, 475-485, doi:10.1016/j.cell.2008.08.031 (2008).

43 Lioy, V. S. et al. Multiscale Structuring of the E. coli Chromosome by NucleoidAssociated and Condensin Proteins. Cell 172, 771-783 e718, doi:10.1016/j.cell.2017.12.027 (2018).

44 Nolivos, S. et al. MatP regulates the coordinated action of topoisomerase IV and MukBEF in chromosome segregation. Nature communications 7, 10466, doi:10.1038/ncomms10466 (2016).

45 Niki, H., Jaffe, A., Imamura, R., Ogura, T. \& Hiraga, S. The new gene mukB codes for a $177 \mathrm{kd}$ protein with coiled-coil domains involved in chromosome partitioning of $\mathrm{E}$. coli. EMBO J 10, 183-193 (1991).

46 Duderstadt, K. E. \& Berger, J. M. AAA+ ATPases in the initiation of DNA replication. Crit Rev Biochem Mol Biol 43, 163-187 (2008).

47 Wolanski, M., Donczew, R., Zawilak-Pawlik, A. \& Zakrzewska-Czerwinska, J. oriCencoded instructions for the initiation of bacterial chromosome replication. Frontiers in microbiology 5, 735, doi:10.3389/fmicb.2014.00735 (2014).

48 Grimwade, J. E. et al. Origin recognition is the predominant role for DnaA-ATP in initiation of chromosome replication. Nucleic acids research 46, 6140-6151, doi:10.1093/nar/gky457 (2018).

49 McGarry, K. C., Ryan, V. T., Grimwade, J. E. \& Leonard, A. C. Two discriminatory binding sites in the Escherichia coli replication origin are required for DNA strand opening by initiator DnaA-ATP. Proceedings of the National Academy of Sciences of the United States of America 101, 2811-2816, doi:10.1073/pnas.0400340101 (2004).

50 Kawakami, H., Keyamura, K. \& Katayama, T. Formation of an ATP-DnaA-specific initiation complex requires DnaA Arginine 285, a conserved motif in the AAA+ protein family. The Journal of biological chemistry 280, 27420-27430, doi:10.1074/jbc.M502764200 (2005).

51 Rozgaja, T. A. et al. Two oppositely oriented arrays of low-affinity recognition sites in oriC guide progressive binding of DnaA during Escherichia coli pre-RC assembly. Mol Microbiol 82, 475-488, doi:10.1111/j.1365-2958.2011.07827.x (2011).

52 Stepankiw, N., Kaidow, A., Boye, E. \& Bates, D. The right half of the Escherichia coli replication origin is not essential for viability, but facilitates multi-forked replication. Mol Microbiol 74, 467-479, doi:10.1111/j.1365-2958.2009.06877.x (2009).

53 Sakiyama, Y., Kasho, K., Noguchi, Y., Kawakami, H. \& Katayama, T. Regulatory dynamics in the ternary DnaA complex for initiation of chromosomal replication in Escherichia coli. Nucleic acids research 45, 12354-12373, doi:10.1093/nar/gkx914 (2017).

54 Kowalski, D. \& Eddy, M. J. The DNA unwinding element: a novel, cis-acting component that facilitates opening of the Escherichia coli replication origin. EMBO J 8, 4335-4344 (1989). 
55 Kaur, G. et al. Building the bacterial orisome: high-affinity DnaA recognition plays a role in setting the conformation of oriC DNA. Mol Microbiol 91, 1148-1163, doi:10.1111/mmi.12525 (2014).

56 Kurokawa, K., Nishida, S., Emoto, A., Sekimizu, K. \& Katayama, T. Replication cyclecoordinated change of the adenine nucleotide-bound forms of DnaA protein in Escherichia coli. Embo J 18, 6642-6652 (1999).

57 Kato, J. \& Katayama, T. Hda, a novel DnaA-related protein, regulates the replication cycle in Escherichia coli. EMBO J 20, 4253-4262, doi:10.1093/emboj/20.15.4253 (2001).

58 Kasho, K., Tanaka, H., Sakai, R. \& Katayama, T. Cooperative DnaA Binding to the Negatively Supercoiled datA Locus Stimulates DnaA-ATP Hydrolysis. The Journal of biological chemistry 292, 1251-1266, doi:10.1074/jbc.M116.762815 (2017).

59 Fujimitsu, K., Senriuchi, T. \& Katayama, T. Specific genomic sequences of E. coli promote replicational initiation by directly reactivating ADP-DnaA. Genes \& development 23, 1221-1233, doi:10.1101/gad.1775809 (2009).

60 Sekimizu, K. \& Kornberg, A. Cardiolipin activation of dnaA protein, the initiation protein of replication in Escherichia coli. The Journal of biological chemistry 263, 7131-7135 (1988).

61 Flatten, I., Fossum-Raunehaug, S., Taipale, R., Martinsen, S. \& Skarstad, K. The DnaA Protein Is Not the Limiting Factor for Initiation of Replication in Escherichia coli. PLoS Genet 11, e1005276, doi:10.1371/journal.pgen.1005276 (2015).

62 Campbell, J. L. \& Kleckner, N. E. coli oriC and the dnaA gene promoter are sequestered from dam methyltransferase following the passage of the chromosomal replication fork. Cell 62, 967-979 (1990).

63 Nievera, C., Torgue, J. J., Grimwade, J. E. \& Leonard, A. C. SeqA blocking of DnaAoriC interactions ensures staged assembly of the E. coli pre-RC. Mol Cell 24, 581-592, doi:10.1016/j.molcel.2006.09.016 (2006).

64 Leonard, A. C. \& Grimwade, J. E. The orisome: structure and function. Frontiers in microbiology 6, 545, doi:10.3389/fmicb.2015.00545 (2015).

65 Hansen, F. G. \& Atlung, T. The DnaA Tale. Frontiers in microbiology 9, 319, doi:10.3389/fmicb.2018.00319 (2018).

66 Katayama, T., Kasho, K. \& Kawakami, H. The DnaA Cycle in Escherichia coli: Activation, Function and Inactivation of the Initiator Protein. Frontiers in microbiology 8, 2496, doi:10.3389/fmicb.2017.02496 (2017).

67 Skarstad, K. \& Katayama, T. Regulating DNA replication in bacteria. Cold Spring Harb Perspect Biol 5, a012922, doi:10.1101/cshperspect.a012922 (2013).

68 Kurokawa, K. et al. Rapid exchange of bound ADP on the Staphylococcus aureus replication initiation protein DnaA. The Journal of biological chemistry 284, 3420134210, doi:10.1074/jbc.M109.060681 (2009).

69 Scholefield, G. \& Murray, H. YabA and DnaD inhibit helix assembly of the DNA replication initiation protein DnaA. Mol Microbiol 90, 147-159, doi:10.1111/mmi.12353 (2013).

70 Scholefield, G., Errington, J. \& Murray, H. Soj/ParA stalls DNA replication by inhibiting helix formation of the initiator protein DnaA. EMBO $J$ 31, 1542-1555, doi:10.1038/emboj.2012.6 (2012).

71 Collier, J. \& Shapiro, L. Feedback control of DnaA-mediated replication initiation by replisome-associated HdaA protein in Caulobacter. J Bacteriol 191, 5706-5716, doi:10.1128/JB.00525-09 (2009). 
72 Wargachuk, R. \& Marczynski, G. T. The Caulobacter crescentus Homolog of DnaA (HdaA) Also Regulates the Proteolysis of the Replication Initiator Protein DnaA. $J$ Bacteriol 197, 3521-3532, doi:10.1128/JB.00460-15 (2015).

73 Jonas, K., Chen, Y. E. \& Laub, M. T. Modularity of the bacterial cell cycle enables independent spatial and temporal control of DNA replication. Curr Biol 21, 1092-1101, doi:10.1016/j.cub.2011.05.040 (2011).

74 Bastedo, D. P. \& Marczynski, G. T. CtrA response regulator binding to the Caulobacter chromosome replication origin is required during nutrient and antibiotic stress as well as during cell cycle progression. Mol Microbiol 72, 139-154, doi:10.1111/j.13652958.2009.06630.x (2009).

75 Val, M. E., Soler-Bistue, A., Bland, M. J. \& Mazel, D. Management of multipartite genomes: the Vibrio cholerae model. Current opinion in microbiology 22, 120-126, doi:10.1016/j.mib.2014.10.003 (2014).

76 Nordstrom, K. \& Dasgupta, S. Copy-number control of the Escherichia coli chromosome: a plasmidologist's view. EMBO reports 7, 484-489, doi:10.1038/sj.embor.7400681 (2006).

77 Tolmasky, M. \& Alonso, J. C. Plasmids: biology and impact in biotechnology and discovery. (ASM Press, 2015).

78 Rasmussen, T., Jensen, R. B. \& Skovgaard, O. The two chromosomes of Vibrio cholerae are initiated at different time points in the cell cycle. EMBO J 26, 3124-3131, doi:10.1038/sj.emboj.7601747 (2007).

79 Frage, B. et al. Spatiotemporal choreography of chromosome and megaplasmids in the Sinorhizobium meliloti cell cycle. Mol Microbiol 100, 808-823, doi:10.1111/mmi.13351 (2016).

80 Deghelt, M. et al. G1-arrested newborn cells are the predominant infectious form of the pathogen Brucella abortus. Nature communications 5, 4366, doi:10.1038/ncomms5366 (2014).

81 Du, W. L. et al. Orderly Replication and Segregation of the Four Replicons of Burkholderia cenocepacia J2315. PLoS Genet 12, e1006172, doi:10.1371/journal.pgen.1006172 (2016).

82 Baek, J. H. \& Chattoraj, D. K. Chromosome I controls chromosome II replication in Vibrio cholerae. PLoS Genet 10, e1004184, doi:10.1371/journal.pgen.1004184 (2014).

83 Pal, D., Venkova-Canova, T., Srivastava, P. \& Chattoraj, D. K. Multipartite regulation of $\mathrm{rctB}$, the replication initiator gene of Vibrio cholerae chromosome II. J Bacteriol 187, 7167-7175, doi:10.1128/JB.187.21.7167-7175.2005 (2005).

84 Duigou, S. et al. Independent control of replication initiation of the two Vibrio cholerae chromosomes by DnaA and RctB. J Bacteriol 188, 6419-6424, doi:10.1128/JB.0056506 (2006).

85 Val, M. E. et al. A checkpoint control orchestrates the replication of the two chromosomes of Vibrio cholerae. Sci Adv 2, e1501914, doi:10.1126/sciadv.1501914 (2016).

86 Griese, M., Lange, C. \& Soppa, J. Ploidy in cyanobacteria. FEMS Microbiol Lett 323, 124-131, doi:10.1111/j.1574-6968.2011.02368.x (2011).

87 Ohbayashi, R. et al. DNA replication depends on photosynthetic electron transport in cyanobacteria. FEMS Microbiol Lett 344, 138-144, doi:10.1111/1574-6968.12166 (2013).

88 Watanabe, S. et al. Light-dependent and asynchronous replication of cyanobacterial multi-copy chromosomes. Mol Microbiol 83, 856-865 (2012).

89 Chen, A. H., Afonso, B., Silver, P. A. \& Savage, D. F. Spatial and temporal organization of chromosome duplication and segregation in the cyanobacterium 
Synechococcus elongatus PCC 7942. PloS one 7, e47837, doi:10.1371/journal.pone.0047837 (2012).

90 Jain, I. H., Vijayan, V. \& O'Shea, E. K. Spatial ordering of chromosomes enhances the fidelity of chromosome partitioning in cyanobacteria. Proceedings of the National Academy of Sciences of the United States of America 109, 13638-13643, doi:10.1073/pnas.1211144109 (2012).

91 Paijmans, J., Bosman, M., Ten Wolde, P. R. \& Lubensky, D. K. Discrete gene replication events drive coupling between the cell cycle and circadian clocks. Proceedings of the National Academy of Sciences of the United States of America 113, 4063-4068, doi:10.1073/pnas.1507291113 (2016).

92 Mendell, J. E., Clements, K. D., Choat, J. H. \& Angert, E. R. Extreme polyploidy in a large bacterium. Proceedings of the National Academy of Sciences of the United States of America 105, 6730-6734, doi:10.1073/pnas.0707522105 (2008).

93 Kornberg, A. \& Baker, T. DNA replication. 2nd edn, (Freeman, 1992).

94 Trojanowski, D. et al. Choreography of the Mycobacterium replication machinery during the cell cycle. MBio 6, e02125-02114, doi:10.1128/mBio.02125-14 (2015).

95 Churchward, G. \& Bremer, H. Determination of deoxyribonucleic acid replication time in exponentially growing Escherichia coli B/r. J Bacteriol 130, 1206-1213 (1977).

96 Allman, R., Schjerven, T. \& Boye, E. Cell cycle parameters of Escherichia coli K-12. J Bacteriol 173, 7970-7974 (1991).

97 Lewis, J. S., Jergic, S. \& Dixon, N. E. The E. coli DNA Replication Fork. Enzymes 39, 31-88, doi:10.1016/bs.enz.2016.04.001 (2016).

98 Beattie, T. R. \& Reyes-Lamothe, R. A Replisome's journey through the bacterial chromosome. Frontiers in microbiology 6, 562, doi:10.3389/fmicb.2015.00562 (2015).

99 Beattie, T. R. et al. Frequent exchange of the DNA polymerase during bacterial chromosome replication. Elife 6, doi:10.7554/eLife.21763 (2017).

100 Lewis, J. S. et al. Single-molecule visualization of fast polymerase turnover in the bacterial replisome. Elife 6, doi:10.7554/eLife.23932 (2017).

101 Liao, Y., Li, Y., Schroeder, J. W., Simmons, L. A. \& Biteen, J. S. Single-Molecule DNA Polymerase Dynamics at a Bacterial Replisome in Live Cells. Biophysical journal 111, 2562-2569, doi:10.1016/j.bpj.2016.11.006 (2016).

102 Robinson, A., Causer, R. J. \& Dixon, N. E. Architecture and conservation of the bacterial DNA replication machinery, an underexploited drug target. Curr Drug Targets 13, 352-372 (2012).

103 Zaritsky, A. \& Pritchard, R. H. Changes in cell size and shape associated with changes in the replication time of the chromosome of Escherichia coli. J Bacteriol 114, 824-837 (1973).

104 Odsbu, I., Morigen \& Skarstad, K. A reduction in ribonucleotide reductase activity slows down the chromosome replication fork but does not change its localization. PloS one 4, e7617, doi:10.1371/journal.pone.0007617 (2009).

105 Zhu, M. et al. Manipulating the Bacterial Cell Cycle and Cell Size by Titrating the Expression of Ribonucleotide Reductase. MBio 8, doi:10.1128/mBio.01741-17 (2017).

106 Olliver, A., Saggioro, C., Herrick, J. \& Sclavi, B. DnaA-ATP acts as a molecular switch to control levels of ribonucleotide reductase expression in Escherichia coli. Mol Microbiol 76, 1555-1571, doi:10.1111/j.1365-2958.2010.07185.x (2010).

107 Sun, L. \& Fuchs, J. A. Escherichia coli ribonucleotide reductase expression is cell cycle regulated. Mol Biol Cell 3, 1095-1105, doi:10.1091/mbc.3.10.1095 (1992).

108 Hanke, P. D. \& Fuchs, J. A. Requirement of protein synthesis for the induction of ribonucleoside diphosphate reductase mRNA in Escherichia coli. Mol Gen Genet 193, 327-331 (1984). 
109 Gon, S. et al. A novel regulatory mechanism couples deoxyribonucleotide synthesis and DNA replication in Escherichia coli. EMBO J 25, 1137-1147, doi:10.1038/sj.emboj.7600990 (2006).

110 Babu, V. M. P., Itsko, M., Baxter, J. C., Schaaper, R. M. \& Sutton, M. D. Insufficient levels of the nrdAB-encoded ribonucleotide reductase underlie the severe growth defect of the Deltahda E. coli strain. Mol Microbiol 104, 377-399, doi:10.1111/mmi.13632 (2017).

111 Torrents, E. et al. NrdR controls differential expression of the Escherichia coli ribonucleotide reductase genes. J Bacteriol 189, 5012-5021, doi:10.1128/JB.00440-07 (2007).

112 McKethan, B. L. \& Spiro, S. Cooperative and allosterically controlled nucleotide binding regulates the DNA binding activity of NrdR. Mol Microbiol 90, 278-289, doi:10.1111/mmi.12364 (2013).

113 Denapoli, J., Tehranchi, A. K. \& Wang, J. D. Dose-dependent reduction of replication elongation rate by (p)ppGpp in Escherichia coli and Bacillus subtilis. Mol Microbiol 88, 93-104, doi:10.1111/mmi.12172 (2013).

114 Wang, J. D., Sanders, G. M. \& Grossman, A. D. Nutritional control of elongation of DNA replication by (p)ppGpp. Cell 128, 865-875, doi:10.1016/j.cell.2006.12.043 (2007).

115 Christensen, R. B., Christensen, J. R., Koenig, I. \& Lawrence, C. W. Untargeted mutagenesis induced by UV in the lacI gene of Escherichia coli. Mol Gen Genet 201, 30-34 (1985).

116 Wendel, B. M., Cole, J. M., Courcelle, C. T. \& Courcelle, J. SbcC-SbcD and ExoI process convergent forks to complete chromosome replication. Proceedings of the National Academy of Sciences of the United States of America 115, 349-354, doi:10.1073/pnas.1715960114 (2018).

117 Midgley-Smith, S. L. et al. Chromosomal over-replication in Escherichia coli recG cells is triggered by replication fork fusion and amplified if replichore symmetry is disturbed. Nucleic acids research 46, 7701-7715, doi:10.1093/nar/gky566 (2018).

118 Sinha, A. K. et al. Division-induced DNA double strand breaks in the chromosome terminus region of Escherichia coli lacking RecBCD DNA repair enzyme. PLoS Genet 13, e1006895, doi:10.1371/journal.pgen.1006895 (2017).

119 Wang, X., Montero Llopis, P. \& Rudner, D. Z. Organization and segregation of bacterial chromosomes. Nature reviews. Genetics 14, 191-203, doi:10.1038/nrg3375 (2013).

120 Adams, D. W., Wu, L. J. \& Errington, J. Cell cycle regulation by the bacterial nucleoid. Current opinion in microbiology 22, 94-101, doi:10.1016/j.mib.2014.09.020 (2014).

121 Postow, L., Crisona, N. J., Peter, B. J., Hardy, C. D. \& Cozzarelli, N. R. Topological challenges to DNA replication: conformations at the fork. Proceedings of the National Academy of Sciences of the United States of America 98, 8219-8226 (2001).

122 Wang, X., Reyes-Lamothe, R. \& Sherratt, D. J. Modulation of Escherichia coli sister chromosome cohesion by topoisomerase IV. Genes \& development 22, 2426-2433 (2008).

123 Joshi, M. C. et al. Regulation of sister chromosome cohesion by the replication fork tracking protein SeqA. PLoS Genet 9, e1003673, doi:10.1371/journal.pgen.1003673 (2013).

124 Barre, F. X. \& Sherratt, D. in The bacterial chromosome (ed N. P. Higgins) Ch. 28, 513-524 (ASM Press, 2005). 
125 Ip, S. C., Bregu, M., Barre, F. X. \& Sherratt, D. J. Decatenation of DNA circles by FtsK-dependent Xer site-specific recombination. EMBO J 22, 6399-6407, doi:10.1093/emboj/cdg589 (2003).

126 Grainge, I. et al. Unlinking chromosome catenanes in vivo by site-specific recombination. Embo J 26, 4228-4238 (2007).

127 Jun, S. \& Wright, A. Entropy as the driver of chromosome segregation. Nature reviews 8, 600-607, doi:10.1038/nrmicro2391 (2010).

128 Kennedy, S. P., Chevalier, F. \& Barre, F. X. Delayed activation of Xer recombination at dif by FtsK during septum assembly in Escherichia coli. Mol Microbiol 68, 10181028, doi:10.1111/j.1365-2958.2008.06212.x (2008).

129 Hobbie, J. E. \& Hobbie, E. A. Microbes in nature are limited by carbon and energy: the starving-survival lifestyle in soil and consequences for estimating microbial rates. Frontiers in microbiology 4, 324, doi:10.3389/fmicb.2013.00324 (2013).

130 Slager, J., Kjos, M., Attaiech, L. \& Veening, J. W. Antibiotic-induced replication stress triggers bacterial competence by increasing gene dosage near the origin. Cell 157, 395406, doi:10.1016/j.cell.2014.01.068 (2014).

131 Ohbayashi, R. et al. Diversification of DnaA dependency for DNA replication in cyanobacterial evolution. ISME J 10, 1113-1121, doi:10.1038/ismej.2015.194 (2016).

132 Errington, J. L-form bacteria, cell walls and the origins of life. Open biology 3, 120143, doi:10.1098/rsob.120143 (2013).

133 Skarstad, K., Steen, H. B. \& Boye, E. Cell cycle parameters of slowly growing Escherichia coli B/r studied by flow cytometry. J Bacteriol 154, 656-662 (1983).

134 Skarstad, K., Steen, H. B. \& Boye, E. Escherichia coli DNA distributions measured by flow cytometry and compared with theoretical computer simulations. J Bacteriol 163, 661-668 (1985).

135 Marczynski, G. T., Dingwall, A. \& Shapiro, L. Plasmid and chromosomal DNA replication and partitioning during the Caulobacter crescentus cell cycle. J Mol Biol 212, 709-722, doi:10.1016/0022-2836(90)90232-B (1990).

136 Le, T. B., Imakaev, M. V., Mirny, L. A. \& Laub, M. T. High-resolution mapping of the spatial organization of a bacterial chromosome. Science 342, 731-734, doi:10.1126/science.1242059 (2013).

137 Potvin-Trottier, L., Luro, S. \& Paulsson, J. Microfluidics and single-cell microscopy to study stochastic processes in bacteria. Current opinion in microbiology 43, 186-192, doi:10.1016/j.mib.2017.12.004 (2018).

138 Ullman, G. et al. High-throughput gene expression analysis at the level of single proteins using a microfluidic turbidostat and automated cell tracking. Philos Trans $R$ Soc Lond B Biol Sci 368, 20120025, doi:10.1098/rstb.2012.0025 (2013).

139 Uphoff, S. et al. Stochastic activation of a DNA damage response causes cell-to-cell mutation rate variation. Science 351, 1094-1097, doi:10.1126/science.aac9786 (2016).

140 Liu, J. et al. Coupling between distant biofilms and emergence of nutrient time-sharing. Science 356, 638-642, doi:10.1126/science.aah4204 (2017).

141 Din, M. O. et al. Synchronized cycles of bacterial lysis for in vivo delivery. Nature 536, 81-85, doi:10.1038/nature18930 (2016).

142 Massalha, H., Korenblum, E., Malitsky, S., Shapiro, O. H. \& Aharoni, A. Live imaging of root-bacteria interactions in a microfluidics setup. Proceedings of the National Academy of Sciences of the United States of America 114, 4549-4554, doi:10.1073/pnas.1618584114 (2017).

143 Walsh, E. J. et al. Microfluidics with fluid walls. Nature communications 8, 816, doi:10.1038/s41467-017-00846-4 (2017). 
144 Lawson, M. J. et al. In situ genotyping of a pooled strain library after characterizing complex phenotypes. Mol Syst Biol 13, 947, doi:10.15252/msb.20177951 (2017).

145 Campos, M. et al. Genomewide phenotypic analysis of growth, cell morphogenesis, and cell cycle events in Escherichia coli. Mol Syst Biol 14, e7573, doi:10.15252/msb.20177573 (2018).

146 Helmstetter, C. E. DNA synthesis during the division cycle of rapidly growing Escherichia coli B/r. J Mol Biol 31, 507-518 (1968).

147 Skarstad, K., Boye, E. \& Steen, H. B. Timing of initiation of chromosome replication in individual Escherichia coli cells. EMBO J 5, 1711-1717 (1986).

148 Michelsen, O., Teixeira de Mattos, M. J., Jensen, P. R. \& Hansen, F. G. Precise determinations of $\mathrm{C}$ and $\mathrm{D}$ periods by flow cytometry in Escherichia coli $\mathrm{K}-12$ and B/r. Microbiology 149, 1001-1010, doi:10.1099/mic.0.26058-0 (2003).

149 Reyes-Lamothe, R., Possoz, C., Danilova, O. \& Sherratt, D. J. Independent positioning and action of Escherichia coli replisomes in live cells. Cell 133, 90-102 (2008).

150 Jensen, R. B., Wang S.C., Shapiro L. A moving DNA replication factory in Caulobacter crescentus. Embo J 20, 4952-4963 (2001).

151 Cook, P. R. The organization of replication and transcription. Science 284, 1790-1795 (1999).

152 Lemon, K. P. \& Grossman, A. D. Localization of bacterial DNA polymerase: evidence for a factory model of replication. Science 282, 1516-1519 (1998).

153 Mangiameli, S. M., Veit, B. T., Merrikh, H. \& Wiggins, P. A. The Replisomes Remain Spatially Proximal throughout the Cell Cycle in Bacteria. PLoS Genet 13, e1006582, doi:10.1371/journal.pgen.1006582 (2017).

154 Reyes-Lamothe, R., Sherratt, D. J. \& Leake, M. C. Stoichiometry and architecture of active DNA replication machinery in Escherichia coli. Science 328, 498-501, doi:328/5977/498 [pii]

10.1126/science.1185757 (2010).

155 Arias-Cartin, R. et al. Replication fork passage drives asymmetric dynamics of a critical nucleoid-associated protein in Caulobacter. EMBO $J$ 36, 301-318, doi:10.15252/embj.201695513 (2017).

156 Breier, A. M., Weier, H.-U. G. \& Cozzarelli, N. R. Independence of replisomes in Escherichia coli chromosomal replication. Proceedings of the National Academy of Sciences of the United States of America 102, 3942-3947 (2005).

157 Bates, D. \& Kleckner, N. Chromosome and replisome dynamics in E. coli: loss of sister cohesion triggers global chromosome movement and mediates chromosome segregation. Cell 121, 899-911 (2005). 
Figure 1 | Stages of the cell cycle and its relation to growth rate. a| During slow growth, the generation time is longer than the $C+D$ periods, resulting in complete replication from initiation to termination occurring in a single cell generation ${ }^{133} . \mathbf{b} \mid$ In fast growth, the generation time is shorter than the $C+D$ periods, resulting in multiple DNA replication initiation events and overlapping replication cycles in each cell; in this case, multiple replication origins initiate replication synchronously ${ }^{17,134}$. c| In species that have a developmental program, as in C. crescentus, only one of type of the differentiate cells is competent for DNA replication. Therefore, initiation of DNA replication is only initiated under suitable growth conditions ${ }^{135}$.

Figure 2 | Chromosome and nucleoid organization. A diagram of the chromosomal structure for two bacteria capable of fast growth (top). Red and blue halves of the circle represent the two replichores. Green arrows starting at the replication origin represent the direction of the replication forks. Relative position and orientation of relevant genes and short sequences is shown. Organization of the DNA molecule requires the formation of DNA loops, which then occupy predictable positions in the cell (bottom left). Four macrodomains, and two unstructured regions (in grey), are depicted. In characterized bacteria, two possible arrangements of the replichores in the nucleoid have been observed (bottom right). In the first case, replichores diverge from each other, occupying different halves of the nucleoid. In the second, they arrange so that they are parallel to each other along the long axis of the cell. For simplicity, the proposed 3D arrangement described in studies that use chromosome conformation capture methods ${ }^{43,136}$ is not depicted.

Figure 3 | Control of initiation of DNA replication. a| DNA replication initiation occurs at a fixed cell volume/origin, with multiple replication origins initiating replication synchronously. This is accompanied by the observation that the ratio of DnaA-ATP / DnaA-ADP fluctuates during the cell cycle, peaking at initiation and dropping shortly afterwards. Red dots in the plots represent the state of DnaA in the cell diagram shown to the left, respectively. The link between these two observations is the key to understanding the timing of initiation. $\mathbf{b} \mid \ln E$. coli, high concentrations of DnaA-ATP are needed to form DnaA-ATP filaments that will fully occupy oriC, which in turn is needed for DNA melting. Multiple mechanisms contribute to vary the concentration of DnaA-ATP in the cell, in the diagram only two of them are depicted as example. The Hda protein bound to the $\beta$-clamp promotes ATP hydrolysis in DnaA-ATP (left). Binding of DnaA-ADP to the DARS sites leads to the exchange of ADP for ATP. c| In B. subtilis, filament formation and full occupancy of oriC are also needed for initiation. However, DnaA filament formation is regulated through direct interactions with other proteins. Three proteins - DnaD, YabA, and Soj - inhibit the formation of DnaA filaments in this organism. $\mathbf{d}$ | Known bacteria carrying multiple chromosomes initiate DNA replication in sequence, from the largest to the smallest starting with the primary chromosome. e| S. elongatus, which is polyploid, initiates DNA replication asynchronously, but it maintains a linear relation between the number of chromosomes and cell volume.

Figure 4 | Regulation of the rate of synthesis of DNA. a| In E. coli, DNA synthesis is catalysed by DNA Pol III coupled to a ring-shaped processivity factor, the $\beta$-clamp. Simultaneous polymerization of both strands is facilitated by the interaction of up to 3 copies of Pol III with the heteropentameric clamp loader. Lagging strand synthesis is initiated at short RNA 
primers, periodically synthesized by the primase, and requires loading of the $\beta$-clamp by the clamp loader at each primer. Single-strand DNA (ssDNA), which accumulates at the lagging strand, is covered by the single strand-binding protein SSB. SSB functions to protect and to prevent formation of secondary structures on the SSDNA. $\mathbf{b}$ | Rate of DNA replication is largely controlled by the concentration of RNR. This in turn is controlled through transcription, with DnaA-ATP acting as both a transcription activator and repressor, depending of its concentration in the cell, and linking the RNR levels to the rate of DNA replication. In addition, the transcription factor NrdR acts as a repressor, binding to the promoter at low concentrations of ATP or dATP. c| Inhibition of primase is a second mechanism for regulation of the rate of DNA replication. The secondary messengers ppGpp and pppGpp repress primase activity.

\section{Box 1 | Tools for single-cell studies of the cell cycle}

The exploitation of single-cell and single-molecule imaging avoids the compromises of ensemble averaging and is revealing major insight into the behaviour of individual cells and the molecular machines that act within them. The ability to grow and analyse individual cells over many generations in microfluidic devices reveals much more information than bulk averages, because the nature of the measured distributions of a given observable reflects the underlying mechanisms of the processes under investigation, while following the changes in observables over time gives further invaluable information on the underlying processes $22,137-$ 139. For example, cells in a population may have two alternative states, 'process on' or 'process off'. These states can be revealed and analysed in individual cells over time, but would be unresolved in bulk studies. Similarly, two different processes can have the same distribution of states (or molecule numbers, or reaction rates), but fluctuate on different timescales, underpinning the value of high temporal resolution studies ${ }^{137}$. Furthermore, adaptations of these methods are being used to follow non-steady state growth in colonies, biofilms and mixed communities ${ }^{140-143}$. At the same time, improved genetic methods and the ability to rapidly activate or inactivate specific molecular processes are allowing the researcher to move from simple observation, to analysis under perturbed conditions ${ }^{144}$. Improvements in highthroughput quantitative imaging, single-molecule tracking and in image analysis are also having important impacts on the quality of data. Genomics is also making important contributions, particularly when combined with single-cell analysis ${ }^{43}$. What is still missing in bacterial systems is the ability to apply chromosome conformation capture ( $\mathrm{Hi}-\mathrm{C})$ and ChIPSeq to single cells at different stages of their cell cycles. Analysis of large data sets, whether they be cell-based, or macromolecule- or gene-based, requires powerful bioinformatic analytical regimes that may utilize machine learning algorithms; this is particularly important in the analysis of large data-set automated cell imaging analysis (eg ref. ${ }^{145}$ ).

\section{Box 2 | Measuring cell cycle parameters: to mother-machine from baby-machine}

Since the birth of molecular biology, scientists have addressed how bacterial generation times relate to the time to replicate a whole chromosome ( $C$ period), the period between replication termination of a chromosome to the subsequent completion of cell division (D period), and any period between birth to the initiation of DNA replication (B period) ${ }^{1,13}$. Cells with overlapping replication cycles that are born with replicating chromosomes have no $B$ period (FIG. 1b). These studies very much focussed on $E$. coli, partly because it is readily manipulable for synchronisation, DNA labelling and perturbation studies. In addition, it was clear from early work that the time to replicate a complete $E$. coli chromosome can be much 
longer than the generation time, therefore demanding regulatory controls in which initiation of DNA replication must occur on chromosomes that have not completed replication. Ensemble studies of synchronised bacteria, or the analysis of chromosomes in single bacteria using flow cytometry or microscopy techniques are required to determine the values of B, C and D periods. In the 1960s, exploitation of a membrane elution technique in which new born E. coli cells are released from progenitor cells bound to a membrane (the baby-machine) enabled the first detailed studies of synchronous populations of cells as they passed through their cell cycle from birth ${ }^{146}$. Pulse-labelling of DNA allowed the C-period to be measured in relation to other cell cycle parameters. Complementary modelling ${ }^{13}$ provided much of the framework for subsequent bacterial cell cycle studies. Then in the 1980s, adaptation of flow cytometry for bacterial cultures allowed the analysis DNA content as a function of cell size ${ }^{147}$. Typically, the DNA content patterns in steady state cells, which have a mixture of replicating and non-replicating chromosomes, are deconvolved. At the same time an aliquot of cells is subjected to 'run-out', in which drug treatment allows completion of DNA replication in division-inhibited cells, thereby giving cells with an integral number of chromosomes, determined by the number of replication origins present at the time of drug addition. This type of approach revolutionised the determination and analysis of cell cycle parameters in those bacteria amenable to flow cytometry analysis (for example, $\left.{ }^{148}\right)$. Subsequently, the ability to label genetic loci and the replisome in live cells growing on agarose pads $35,36,122,149,150$, or in microfluidic devices, has enabled direct measurement of $B$, $C$ and $D$ periods in cells, along with the periods of cohesion/catenation between newly replicated chromosomes (Box 1). Now, the use of microfluidic mother machines, accompanied by replisome and genetic locus labelling, is providing unprecedented insight into cell cycle parameters and their variance over many tens of generations, both in steady state and perturbed conditions ${ }^{22,130}$.

\section{Box. 3 |Does DNA replication occur in factories?}

The replication factory model posits that a discrete cellular structure organises the activity of multiple replisomes contained within it ${ }^{151}$. The model gained acceptance in bacteria after multiple copies of fluorescently labelled replisome were shown to co-localize in $B$. subtilis 152. A similar spatial concentration containing two or more copies of the replisome has been shown for other bacteria ${ }^{79,89,150}$, and concentration of replisomes into a 'factory-like' organization, where sister replisomes co-localize frequently (but not exclusively), have been proposed for $E$. coli ${ }^{153}$. But does co-localization assayed by light microscopy demonstrate a physical (and coordinated) link between replisomes? In our opinion it does not. Even if multiple replisomes are present in a diffraction-limited spot, individual replisomes could be tens to hundreds of nanometers away from each other, far from the few-nanometer distances that characterize interacting proteins. Furthermore, the vast majority of data suggest that replisomes can act independently of each other. Indeed, time-lapse microscopy have showed that replisomes in E. coli can act for long periods of time at distant parts in the cell, even though their localization over time is variable ${ }^{149}$. Quantitative imaging showed that a single replisome 'spot' had stoichiometries only consistent with a single replisome being present in that spot ${ }^{154}$. Separation of sister replisomes is much clearer in cells unable to segregate their chromosomes ${ }^{122}$. Furthermore, even in species with close localization between sister replisomes, they are also known to separate transiently by hundreds of nanometres ${ }^{8,155}$. There is also no evidence of a functional role for replisomes to be concentrated into factories. The activity of a replisome is not influenced by its sister that 
originated from the same initiation event ${ }^{156}$. And given that segregation of chromosomal loci occurs minutes after their replication, the hypothesis that DNA replication provides a force for DNA segregation seems untenable ${ }^{36,149,157}$. How then can co-localization of replisomes be explained? We favour the idea that local high concentration of replisomes has more to do with how the parental chromosome and the newly replicated sisters are organised during DNA replication, rather than implying a cellular structure that physically links multiple replisomes. Therefore, the position of replisomes can be explained by small cell volumes, relative movement of newly replicated DNA with respect to the unreplicated DNA, and limited optical resolution (See accompanying figure; topo IVts indicates cells where chromosome segregation does not occur). 


\section{Glossary}

Generation time_average time needed for a newly born cell to divide.

Replication origin_sequence on a chromosome or plasmid where DNA replication initiates.

Replisome_multi-subunit protein complex that carries out DNA replication.

Replication fork_the Y-shape DNA structure formed at the point where DNA is unwound during DNA replication.

Ter sites_sequence that stalls the progression of the replication fork in an orientationdependent manner

KOPS _ sequence that modulates the direction of translocation of the motor protein FtsK on DNA

Chi sequences_sites that modulate the activity of the protein complex RecBCD during recombination

Nucleoid_Cell body that contains the chromosomal DNA and DNA-binding proteins

Replichores_the two chromosomal halves starting at the replication origin, each replicated by a different replication fork

SMC complex_Structural Maintenance of Chromosomes.

NAPs_Nucleoid Associated Proteins

B-period_period from cell birth to initiation of DNA replication

C-period_period from initiation to termination of DNA replication

D-period_period from the end of DNA replication to cell division

Cohesion time_time from the replication of a locus to the separation of the newly replicated sister loci

Swarmer cell_the mobile developmental state in C. crescentus.

Stalked cell_the sessile developmental state in C. crescentus.

Iteron plasmids_characterized by a replication origin composed of repeated sequences that bind to a cognate initiator protein.

Primase_replisome subunit that synthesizes short RNA primers which can then be elongated by the DNA polymerase

SOS response_cellular response to DNA damage leading to inhibition of cell division and induction of DNA repair systems.

Divisome_Structure that forms around the middle of the cell, composed of multiple proteins required for cell division. 


\section{Acknowledgements}

Research in the Reyes-Lamothe laboratory is funded by the Natural Sciences and Engineering Research Council of Canada (NSERC\# 435521-2013), the Canadian Institutes for Health Research (CIHR MOP\# 142473), and the Canada Research Chairs program. The Sherratt laboratory is funded by a Wellcome Investigator Award (200782/Z/16/Z). We thank many colleagues for stimulating discussions, in particular Stephan Uphoff and Bela Novak (Oxford); and members of the Reyes lab. 